\title{
Rationale and Study Protocol of the J-SAVER Study: A Phase II Study of S-1 on Alternate Days Combined with Bevacizumab in Patients Aged $\geq 75$ Years with Metastatic Colorectal Cancer
}

\author{
Toshikazu Moriwaki1 ${ }^{*}$, Tetsuya Eto², Akihito Tsuji3 ${ }^{3}$, Nobushige Kakinoki', Mitsuo Shimada5, \\ Takashi Maeba', Hiroaki Hatano", Ikuo Takahashi8, Hiroyasu Ishida9, Kazuho Ikeda10, \\ Yoshiaki Bando11, Ichinosuke Hyodo ${ }^{1}$
}

\author{
${ }^{1}$ Division of Gastroenterology, Faculty of Medicine, University of Tsukuba, Tsukuba, Japan \\ ${ }^{2}$ Department of Gastroenterology, Tsuchiura Kyodo General Hospital, Tsuchiura, Japan \\ ${ }^{3}$ Department of Medical Oncology, Kobe City Medical Center General Hospital, Kobe, Japan \\ ${ }^{4}$ Department of Gastroenterology, Hitachi General Hospital, Hitachi, Japan \\ ${ }^{5}$ Department of Surgery, Tokushima University, Tokushima, Japan \\ ${ }^{6}$ Department of Surgery, Japan Community Health Care Organization Ritsurin Hospital, Takamatsu, Japan \\ ${ }^{7}$ Department of Surgery, Matsuyama Shimin Hospital, Matsuyam, Japan \\ ${ }^{8}$ Department of Surgery, Matsuyama Red Cross Hospital, Matsuyama, Japan \\ ${ }^{9}$ Department of Gastroenterology, National Hospital Organization Mito Medical Center, Higashi Ibaraki-Gun, Japan \\ ${ }^{10}$ Department of Gastroenterology, Koyama Memorial Hospital, Kashima, Japan \\ ${ }^{11}$ Department of Surgery, Tokushima Prefecture Naruto Hospital, Naruto, Japan \\ Email: ${ }^{\star}$ tmoriwak@gmail.com
}

How to cite this paper: Moriwaki, T., Eto, T., Tsuji, A., Kakinoki, N., Shimada, M., Maeba, T., Hatano, H., Takahashi, I., Ishida, H., Ikeda, K., Bando, Y. and Hyodo, I. (2017) Rationale and Study Protocol of the J-SAVER Study: A Phase II Study of S-1 on Alternate Days Combined with Bevacizumab in Patients Aged $\geq 75$ Years with Metastatic Colorectal Cancer. Journal of Cancer Therapy, 8, 1040-1048. https://doi.org/10.4236/jct.2017.811088

Received: October 31, 2017

Accepted: November 25, 2017

Published: November 28, 2017

\begin{abstract}
Fluoropyrimidine combined with bevacizumab is commonly used in elderly patients with metastatic colorectal cancer worldwide. However, the proportion of elderly patients who discontinued treatment due to toxicities was higher than that of younger patients. The aim of this study is to develop a less toxic schedule of S-1, while maintaining the anti-tumor effect. This phase II study is aimed to evaluate an alternate-day administration of S-1 combined with bevacizumab in untreated elderly patients (aged $\geq 75$ years) with metastatic colorectal cancer. The primary endpoint is progression-free survival, and the secondary endpoints are safety, response rate, and overall survival. The expected median progression-free survival is 8.5 months, and the minimum efficacy threshold is 5.0 months. The total required sample size is calculated as 50 patients, with a 2 -sided type I error of 0.10 and a power of more than $80 \%$. This study is ongoing, and fifty-four patients were enrolled until October 2016. We hope that S-1 on alter-
\end{abstract}


Copyright $\odot 2017$ by authors and Scientific Research Publishing Inc. This work is licensed under the Creative Commons Attribution International License (CC BY 4.0).

http://creativecommons.org/licenses/by/4.0/ nate days combined with bevacizumab for elderly patients with colorectal cancer is well tolerated and can maintain effectiveness. Trial registration: UMIN clinical trials UMIN000010402.

\section{Keywords}

Alternate Days, Bevacizumab, Colorectal Cancer, Fluoropyrimidine, Elderly, Older, S-1

\section{Introduction}

Colorectal cancer (CRC) is one of the most common causes of cancer-related deaths worldwide [1], and the mortality from CRC among all cancers in Japan is the third highest for men and the highest for women [2]. Among patients with CRC, those aged 75 years and older account for more than $70 \%$ of the total mortality in men and women combined. Chemotherapy opportunities for metastatic CRC (mCRC) in elderly patients are therefore increasing [2]. Doublet [fluoropyrimidine (FP) plus oxaliplatin or irinotecan] chemotherapy combined with a targeted agent (e.g., bevacizumab, cetuximab, or panitumumab) is recognized as the standard treatment for patients with mCRC [3] [4] [5]. Younger patients can tolerate the doublet regimen combined with a targeted agent, whereas elderly patients often cannot tolerate this combination because many of them develop comorbidity and/or functional disorders. Therefore, a combination of FP and bevacizumab is selected when physicians determine that a patient is unable to tolerate doublet therapy with/without a targeted agent or if the patient refuses those regimens. In several previous trials, FP plus bevacizumab significantly improved the progression-free survival (PFS) (8.8 - 9.2 months versus 5.1 - 5.6 months) as well as the overall survival (OS) (15.5 - 20.7 months versus 12.9 - 16.8 months), compared to FP monotherapy [6] [7] [8]. FP plus bevacizumab has been recognized as one of the standard chemotherapies in elderly patients with mCRC.

In Japan, S-1, which is one of the FPs, was also evaluated in two phase II trials for patients with mCRC [9] [10]. The response rate was 39.5\% and $35.5 \%$, and median OS was 11.9 months and 12.0 months, respectively. Based on these results, a phase II trial of S-1 plus bevacizumab was conducted in patients with mCRC aged $\geq 65$ years (BASIC trial) [11]. The schedule consisted of a 6 -week cycle. S-1 was administered orally twice daily for the first 28 days of each cycle, at a dose determined by body surface area (BSA) $\left(<1.25 \mathrm{~m}^{2}, 40 \mathrm{mg} ; 1.25-1.50 \mathrm{~m}^{2}, 50 \mathrm{mg}\right.$; and $>1.50 \mathrm{~m}^{2}, 60 \mathrm{mg}$ ). Bevacizumab at $5 \mathrm{mg} / \mathrm{kg}$ was administered intravenously on day 1, day 15, and day 29 of each cycle. This regimen produced promising results with a response rate, median PFS, and median OS of 43\%, 9.9 months, and 27.5 months, respectively.

S-1 on alternate days was conducted as a new administration schedule [12]. A clear difference in the cell cycles of human normal and tumor cells has been dis- 
covered [13] [14] [15]. The normal cell cycle was as short as approximately 0.5 to 1.5 days, whereas a tumor cell cycle ranged from 3 to 5 days, and the duration of the S-phase that 5-fluorouracil acted predominantly on was $\geq 1$ day in most cancer cells. Based on this difference in cell cycles, a regimen with S-1 administration on alternate days was designed. In a preclinical study, the anticancer activity of S-1 was comparable to or better than that of daily dosing [16]. In a retrospective study, that regimen was conducted in patients with gastric cancer who refused daily dosing of S-1 for 4 weeks followed by 2 weeks of rest, to cope with grade 1 and higher non-hematologic toxicities. The incidence of grade 2 and more non-hematologic toxicities was observed in only $2.8 \%$ of the patients, and median time to treatment failure and median OS were 6 months and 11 months, respectively [17]. Thus, it is expected that S-1 on alternate days is well tolerated and the anti-tumor activity might be equivalent to or better than that of daily dosing of S-1 for elderly patients because the compliance improves.

\section{Patients and Methods}

\subsection{Study Design}

This is a multi-institutional (27 institutions) study by the NPO Tsukuba Cancer Clinical Trial Group (TCTG) and the Shikoku Gastrointestinal Oncology Study Group (SGOSG), prospective, open labeled, single-arm phase II trial (J-SAVER: Joint study of S-1 on Alternate days combined with bevacizumab in Elderly patients with metastatic colorectal cancer) in Japan. Patients must fulfill all the inclusion and exclusion criteria before enrollment in the study. The inclusion and exclusion criteria are listed in Table 1. After confirmation of the eligibility, registration is made via a fax to the Registration Center in the TCTG. Enrolled patients will be started on the study treatment within 14 days from the day of enrollment.

\subsection{Ethical Aspects}

The study will be conducted according to the Declaration of Helsinki/Tokyo and to the Japanese Clinical Research Guidelines. The protocol has been approved by the ethics committee of each participating institution. An informed consent form is signed by all study participants. This study protocol has been registered at the University Hospital Medical Information Network, UMIN000010402, on 2nd April 2013.

\subsection{Treatment Schedule}

The following treatment methods will be repeated until tumor progression, severe adverse events, or patients' refusal. Dose modification will depend upon the toxicities. Patients will receive $40 \mathrm{mg}$ (BSA $\leq 1.25 \mathrm{~m}^{2}$ ), $50 \mathrm{mg}$ (BSA $>1.25$ to $\leq 1.50$ $\mathrm{m}^{2}$ ), or $60 \mathrm{mg}$ (BSA $>1.50 \mathrm{~m}^{2}$ ) of S-1 orally, twice a day, on Monday, Wednesday, Friday, and Sunday every week. Bevacizumab at $7.5 \mathrm{mg} / \mathrm{kg}$ will be administered every 3 weeks (Figure 1 ). 
Table 1. Inclusion and exclusion criteria.

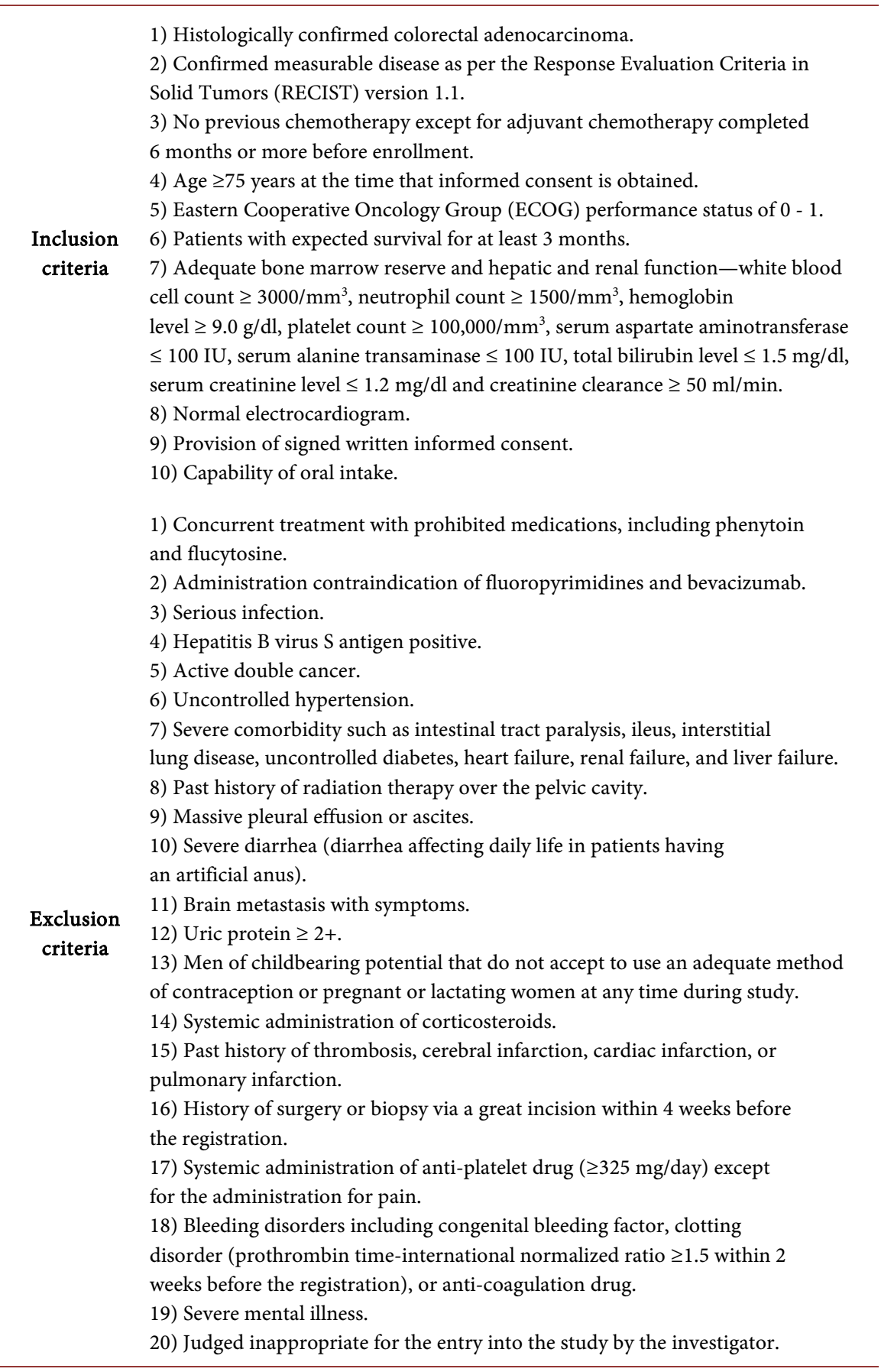

\subsection{Follow-Up}

Patients are assessed according to the Common Terminology Criteria for Adverse Events version 4.0 to detect any adverse events that develop during the treatment. The blood tests include a complete blood cell count, measurement of liver and renal function, and tumor markers (carcinoembryonic antigen and carbohydrate antigen 19 - 9), and the urine test includes uric protein. Observation, assessment, and testing are performed every week until the second administration of 


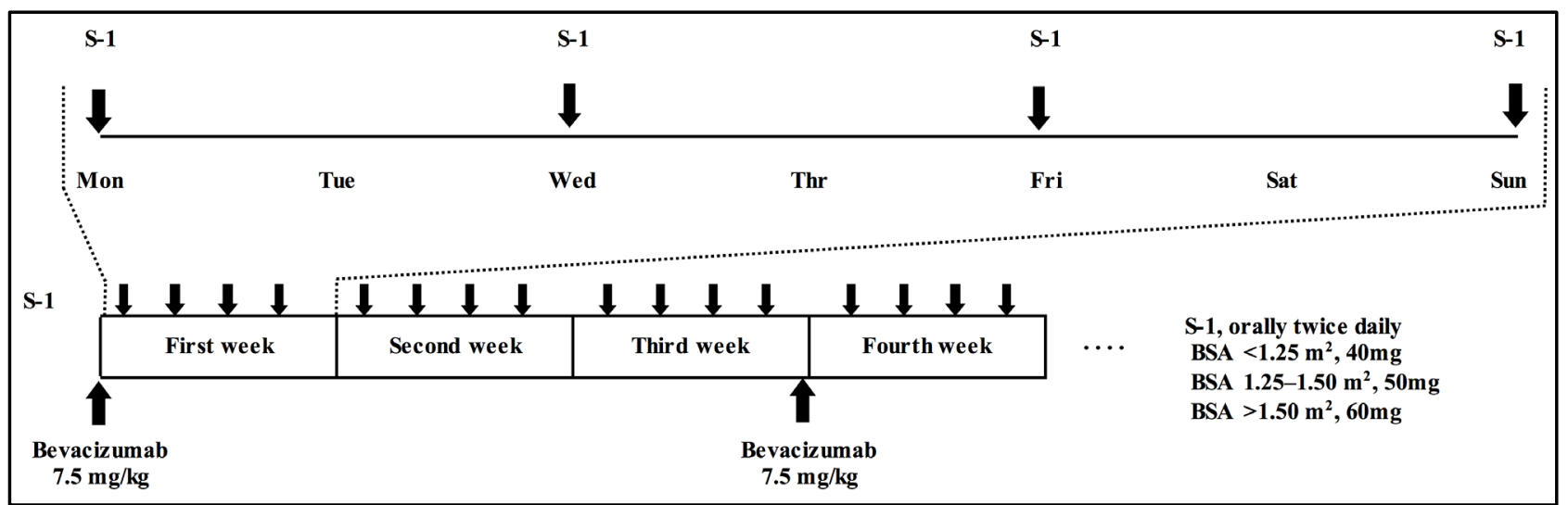

Figure 1. Treatment schedule: BSA — body surface area.

bevacizumab, and at every 3 weeks (the date of administration of bevacizumab) thereafter. Tumor assessments are performed according to RECIST version 1.1. Computed tomography (or magnetic resonance imaging) will be performed every 8 weeks for measurements and evaluation.

\subsection{Statistical Methods}

The primary endpoint is PFS. The secondary endpoints are safety, response rate, and OS. PFS is defined as the time from enrollment till disease progression or death from any cause. OS is defined as the time from enrollment till death from any cause.

In the previous phase II study of S-1 monotherapy, the median PFS was 5.1 months in patients with mCRC [9] [12]. The median PFS of 5-fluorouracil and leucovorin plus bevacizumab therapy was improved by 3.7 months compared with 5-fluorouracil and leucovorin monotherapy in a randomized phase II study [18]. Thus, the expected median PFS is 8.5 months, and the minimum efficacy threshold is 5.0 months. The total required sample size is calculated as 50 patients, with a 2 -sided type I error of 0.10 and a power of $\geq 80 \%$. If the enrollment of patients is fulfilled early on, the total required sample size will be increased to 67 patients. As the result, the accuracy of median PFS is with a 2-sided type I error of 0.05 and a power of $\geq 80 \%$.

\section{Results}

Enrollment started in April 2013. Although the enrollment period was scheduled for two years, it was extended to 3.5 years because of slow accrual. Fifty-four patients were enrolled until October 2016. Of 54 enrolled patients, the following patients were excluded: 1 patient with thrombosis was excluded from this study; 1 patient refused study treatment continuation before the first effective evaluation; 1 patient had only non-target lesion; and 1 patient was found out that was complicated with biliary tract cancer after start of study treatment. Therefore, 50 patients for efficacy and 53 patients for safety will be evaluated (Figure 2).

Patient characteristics list in Table 2. Median age was 79 years (range, 75 - 88 


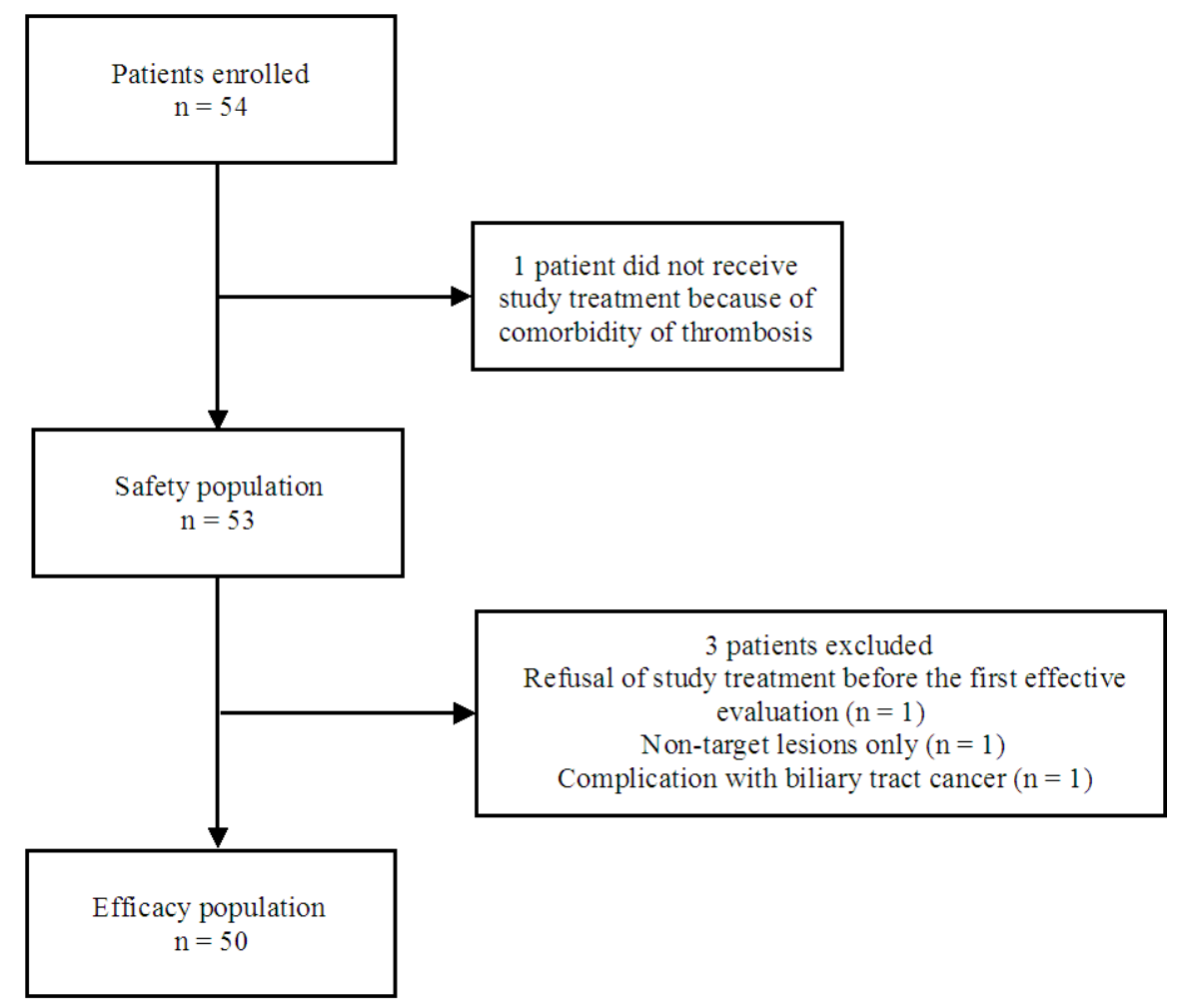

Figure 2. Patients flow.

Table 2. Patient characteristics.

\begin{tabular}{cccc}
\hline Parameter & & $N$ & $\%$ \\
\hline Age, years & Median (range) & $79(75-88)$ \\
Gender & Male & 25 & 50 \\
& Female & 25 & 50 \\
ECOG PS & 0 & 28 & 56 \\
& 1 & 22 & 44 \\
Primary tumor site & Right side & 15 & 30 \\
& Left side & 35 & 70 \\
Surgery on primary tumor & Yes & 39 & 78 \\
& Liver & 28 & 56 \\
& Lung & 19 & 38 \\
Metastatic organ site & Peritoneum & 15 & 30 \\
& Lymph node & 11 & 22 \\
& Others & 12 & 24 \\
& & & 52 \\
& $\mathbf{1}$ & 26 & 34 \\
Number of metastatic organ site & $\mathbf{2}$ & 7 & 14 \\
\hline
\end{tabular}

${ }^{\mathrm{a} C e c u m}$, ascending colon, and transverse colon; ${ }^{\mathrm{b}}$ Descending colon, sigmoid colon, and rectum.

years). ECOG PS was 0 in 28 patients and 1 in 22 patients. Main of metastatic organ was liver in 28 patients, lung in 19 patients, peritoneum in 15 patients, and lymph node in 11 patients. A half of patients had one metastatic organ site. The 
median follow-up time was 34.5 months (range, 25.6 - 44.9 months). The primary endpoint will be analyzed by the end of 2017 .

\section{Discussion}

Although the elderly patients tolerated FP plus bevacizumab well in the trials, the incidence of adverse events did not decrease when compared to the younger patients. In a subgroup analysis by age from a randomized trial of capecitabine, bevacizumab and mitomycin C (AGITG MAX trial), incidences of grade 3 or more diarrhea, hand-foot skin reaction, and fatigue were observed in $21 \%, 27 \%$, and $13 \%$, respectively, in elderly patients who received capecitabine plus bevacizumab, compared to $15 \%, 27 \%$, and $11 \%$, respectively, in the younger patients who received the same regimen [8]. In a randomized trial of capecitabine plus bevacizumab versus capecitabine monotherapy in elderly patients with mCRC (AVEX trial), the incidence of grade 3 or more toxicities was observed in $40 \%$ patients [7]. In the BASIC trial, the incidence of grade 3 or more toxicities was observed in less than $10 \%$ patients; however, $36 \%$ of the patients discontinued treatment due to toxicities [11]. Similar results were observed in a phase II study of uracil-tegafur plus oral leucovorin combined with bevacizumab in elderly patients with mCRC (grade 3 or more toxicities in less than $10 \%$ patients and discontinuation of study treatment due to any of the toxicities in $25 \%$ patients) [19]. A novel FP-containing regimen that is better tolerated should be developed for the elderly patients.

\section{Conclusion}

We hope that S-1 on alternate days combined with bevacizumab for elderly patients with metastatic colorectal cancer is well tolerated and can maintain effectiveness when comparing to FP combined with bevacizumab regimens which are previous reported.

\section{Acknowledgements}

We are grateful to Kentaro Yamazaki, Takako E. Nakajima, and Takehiro Oikawa for their important contributions to this study as part of the Data and Safety Monitoring Committee and Miss Mizuki Aida for data management. We thank the patients, their families, all the investigators who participated in the study. The participating investigators are as follows: Y. Sakai and T. Eto (Tsuchiura Kyodo General Hospital); H. Ishida, S. Yoshida, Y. Ito, and M. Shimoyamada (National Hospital Organization Mito Medical Center); S. Endo (Shinmatsudo Central General Hospital); I. Hyodo, T. Moriwaki, and Y. Yamamoto (University of Tsukuba); H. Kuramochi (Tokyo Women's Medical University Yachiyo Medical Center); M. Sato (Ryugasaki Saiseikai Hospital); A. Tsuji and H. Satake (Kobe City Medical Center General Hospital); Y. Bando, M. Chikakiyo (Tokushima Prefecture Naruto Hospital); T. Maeba, T. Fujita, and S. Mori (JCHO Ritsurin Hospital), K. Ikezawa, A. Soeda, and M. Kobayashi (Tsukuba Memorial Hospital), M. Shimada, J. Higashijima, and T. Nakao (Tokushima University), M. Inukai and Y. Suzuki (Ka- 
gawa University), K. Amagai (Ibaraki Prefectural Central Hospital and Cancer Center), M. Morimoto (National Hospital Organization Ehime Medical Center and Ehime University Graduate School of Medicine), K. Kobayashi (Nagasaki University School of Medicine), N. Kakinoki (Hitachi General Hospital), M. Ando (Anan Kyoei Hospital), H. Hatano (Matsuyama Shimin Hospital), H. Ishii (Saiseikai Saijo Hospital), M. Mizuta (Mitoyo General Hospital), M. Kobayashi (Kochi Medical School), I. Takahashi (Matsuyama Red Cross Hospital), Y. Negoro (Kochi Health Sciences Center), K. Ikeda (Koyama Memorial Hospital), T. Mamiya (Hitachinaka General Hospital), and T. Nishina (National Hospital Organization Shikoku Cancer Center). This work is supported by the NPO Tsukuba Cancer Clinical Trial Group (TCTG).

\section{Conflict of Interest}

The authors declare that they have no conflict of interest regarding the publication of this article.

\section{References}

[1] Ferlay, J., Shin, H.R., Bray, F., Forman, D. and Mathers, C. (2010) Estimates of Worldwide Burden of Cancer in 2008: GLOBOCAN 2008. International Journal of Cancer, 127, 2893-2917. https://doi.org/10.1002/ijc.25516

[2] Matsuda, A., Matsuda, T., Shibata, A., Katanoda, K. and Sobue, T. (2013) Cancer Incidence and Incidence Rates in Japan in 2007: A Study of 21 Population-Based Cancer Registries for the Monitoring of Cancer Incidence in Japan (MCIJ) Project. Japanese Journal of Clinical Oncology, 43, 328-336. https://doi.org/10.1093/jjco/hys233

[3] Saltz, L.B., Clarke, S., Diaz-Rubio, E., Scheithauer, W. and Figer, A. (2008) Bevacizumab in Combination with Oxaliplatin-Based Chemotherapy as First-Line Therapy in Metastatic Colorectal Cancer: A Randomized Phase III Study. Journal of Clinical Oncology, 26, 2013-2019. https://doi.org/10.1200/JCO.2007.14.9930

[4] Van Cutsem, E., Kohne, C.H., Hitre, E., Zaluski, J. and Chang, C.C.R. (2009) Cetuximab and Chemotherapy as Initial Treatment for Metastatic Colorectal Cancer. The New England Journal of Medicine, 360, 1408-1417. https://doi.org/10.1056/NEJMoa0805019

[5] Douillard, J.Y., Siena, S., Cassidy, J., Tabernero, J. and Burkes, R. (2010) Randomized, Phase III trial of Panitumumab with Infusional Fluorouracil, Leucovorin, and Oxaliplatin (FOLFOX4) versus FOLFOX4 Alone as First-Line Treatment in Patients with Previously Untreated Metastatic Colorectal Cancer: The PRIME Study. Journal of Clinical Oncology, 28, 4697-4705. https://doi.org/10.1200/JCO.2009.27.4860

[6] Kabbinavar, F.F., Hambleton, J., Mass, R.D., Hurwitz, H.I. and Bergsland, E. (2005) Combined Analysis of Efficacy: The Addition of Bevacizumab to Fluorouracil/Leucovorin Improves Survival for Patients with Metastatic Colorectal Cancer. Journal of Clinical Oncology, 23, 3706-3712. https://doi.org/10.1200/JCO.2005.00.232

[7] Cunningham, D., Lang, I., Marcuello, E., Lorusso, V. and Ocvirk, J. (2013) Bevacizumab Plus Capecitabine versus Capecitabine Alone in Elderly Patients with Previously Untreated Metastatic Colorectal Cancer (AVEX): An Open-Label, Randomised Phase 3 Trial. The Lancet Oncology, 14, 1077-1085. 
https://doi.org/10.1016/S1470-2045(13)70154-2

[8] Price, T.J., Zannino, D., Wilson, K., Simes, R.J. and Cassidy, J. (2012) Bevacizumab Is Equally Effective and No More Toxic in Elderly Patients with Advanced Colorectal Cancer: A Subgroup Analysis from the AGITG MAX Trial: An International Randomised Controlled Trial of Capecitabine, Bevacizumab and Mitomycin C. Annals of Oncology, 23, 1531-1536. https://doi.org/10.1093/annonc/mdr488

[9] Ohtsu, A., Baba, H., Sakata, Y., Mitachi, Y. and Horikoshi, N. (2000) Phase II Study of S-1, a Novel Oral Fluorophyrimidine Derivative, in Patients with Metastatic Colorectal Carcinoma. S-1 Cooperative Colorectal Carcinoma Study Group. British Journal of Cancer, 83, 141-145.

[10] Shirao, K., Ohtsu, A., Takada, H., Mitachi, Y. and Hirakawa, K. (2004) Phase II Study of Oral S-1 for Treatment of Metastatic Colorectal Carcinoma. Cancer, 100, 2355-2361. https://doi.org/10.1002/cncr.20277

[11] Yoshida, M., Muro, K., Tsuji, A., Hamamoto, Y. and Yoshino, T. (2015) Combination Chemotherapy with Bevacizumab and S-1 for Elderly Patients with Metastatic Colorectal Cancer (BASIC Trial). European Journal of Cancer, 51, 935-941. https://doi.org/10.1016/j.ejca.2015.03.007

[12] Shirasaka, T. (2009) Development History and Concept of an Oral Anticancer Agent S-1 (TS-1): Its Clinical Usefulness and Future Vistas. Japanese Journal of Clinical Oncology, 39, 2-15. https://doi.org/10.1093/jjco/hyn127

[13] Cronkite, E.P., Bond, V.P., Fliedner, T.M. and Rubini, J.R. (1959) The Use of Tritiated Thymidine in the Study of DNS Synthesis and Cell Turnover in Hemopoietic Tissues. Laboratory Investigation, 8, 263-275.

[14] Lipkin, M., Sherlock, P. and Bell, B. (1963) Cell Proliferation Kinetics in the Gastrointestinal Tract of Man. Ii. Cell Renewal in Stomach, Ileum, Colon, and Rectum. Gastroenterology, 45, 721-729.

[15] Clarkson, B., Ota, K., Ohkita, T. and O’Connor, A. (1965) Kinetics of Proliferation of Cancer Cells in Neoplastic Effusions in Man. Cancer, 18, 1189-1213. https://doi.org/10.1002/1097-0142(196510)18:10<1189::AID-CNCR2820181002>3.0 .CO;2-8

[16] Arai, W., Hosoya, Y., Haruta, H., Kurashina, K. and Saito, S. (2008) Comparison of Alternate-Day versus Consecutive-Day Treatment with S-1: Assessment of Tumor Growth Inhibition and Toxicity Reduction in Gastric Cancer Cell Lines in Vitro and in Vivo. International Journal of Clinical Oncology, 13, 515-520. https://doi.org/10.1007/s10147-008-0780-4

[17] Arai, W., Hosoya, Y., Hyodo, M., Yokoyama, T. and Hirashima, Y. (2004) Alternate-Day Oral Therapy with TS-1 for Advanced Gastric Cancer. International Journal of Clinical Oncology, 9, 143-148. https://doi.org/10.1007/s10147-004-0381-9

[18] Kabbinavar, F.F., Schulz, J., McCleod, M., Patel, T. and Hamm, J.T. (2005) Addition of Bevacizumab to Bolus Fluorouracil and Leucovorin in First-Line Metastatic Colorectal Cancer: Results of a Randomized Phase II Trial. Journal of Clinical Oncology, 23, 3697-3705. https://doi.org/10.1200/JCO.2005.05.112

[19] Nishina, T., Moriwaki, T., Shimada, M., Higashijima, J. and Sakai, Y. (2016) Uracil-Tegafur and Oral Leucovorin Combined With Bevacizumab in Elderly Patients (Aged $>/=75$ Years) with Metastatic Colorectal Cancer: A Multicenter, Phase II Trial (Joint Study of Bevacizumab, Oral Leucovorin, and Uracil-Tegafur in Elderly Patients [J-BLUE] Study). Clinical Colorectal Cancer, 15, 236-242. https://doi.org/10.1016/j.clcc.2015.12.001 